\title{
Caracterização de lodo produzido nas lavanderias têxteis da região Agreste de Pernambuco para uso em materiais de construção alternativos
}

\author{
Characterization of the sludge produced in textile laundries in Agreste region of Pernambuco for \\ use in alternative construction materials
}

Caracterización de lodo producido en lavanderías textiles en la región de Agreste de Pernambuco para su uso en materiales de construcción

Recebido: 19/03/2021 | Revisado: 30/03/2021 | Aceito: 05/04/2021 | Publicado: 16/04/2021

Lyneker Souza de Moura

ORCID: https://orcid.org/0000-0002-9343-6916

Universidade Federal de Pernambuco, Brasil

E-mail: lyneker@gmail.com

Carina Lessa Silva

ORCID: https://orcid.org/0000-0003-0789-5436

Universidade Federal de Pernambuco, Brasil

E-mail: carinalessa1999@gmail.com

Ana Carolina dos Santos Reis

ORCID: https://orcid.org/0000-0001-6405-5412

Universidade Federal de Pernambuco, Brasil

E-mail: carolinasreis02@gmail.com

Erika Pinto Marinho

ORCID: https://orcid.org/0000-0001-7405-0877

Universidade Federal de Pernambuco, Brasil

E-mail: erika.pmarinho@ufpe.br

Ana Cecília Vieira da Nóbrega

ORCID: https://orcid.org/0000-0003-1118-1911

Universidade Federal de Pernambuco, Brasil

E-mail: anacecilia.nobrega@ufpe.br

Arnaldo Manoel Pereira Carneiro

ORCID: https://orcid.org/0000-0002-4279-7156

Universidade Federal de Pernambuco, Brasil

E-mail: arnaldo.carneiro@ufpe.br

\begin{abstract}
Resumo
O lodo têxtil é um resíduo resultante do procedimento de tratamento físico-químico do efluente das lavanderias têxteis. Esse material é classificado pela norma brasileira como resíduo não perigoso, tendo em vista que, apesar da sua variada composição química, seus componentes não apresentam grandes riscos de contaminação humana. Diante do descarte inadequado desse resíduo na região do Agreste pernambucano pelas lavanderias, percebeu-se a necessidade da geração de alternativas que proporcionem o uso desse material, uma vez que atualmente não há relatos acerca da sua reciclagem ou aproveitamento. O objetivo deste trabalho foi constatar que os materiais de construção são uma excelente matriz receptora desse resíduo, proporcionando uma redução considerável de seu descarte inadequado e dos possíveis danos ambientais. Para viabilizar o uso desse resíduo, o lodo foi caracterizado por meio de análises de massa específica, granulometria a laser, análise termogravimétrica e térmica diferencial, fluorescência de raios-X, difração de raios-X, microscopia eletrônica de varredura, espectroscopia no infravermelho por transformada de Fourier. Foi obtido um valor de $2,20 \mathrm{~g} / \mathrm{cm}^{3}$ para sua massa específica e um diâmetro médio de partículas de $26,10 \pm 0,65 \mu \mathrm{m}$, sendo $97,15 \%$ delas com diâmetro inferior a $80 \mu \mathrm{m}$, o que caracterizou o material como fíler. Quanto à sua composição, os principais componentes determinados foram $\mathrm{CaO}, \mathrm{MgO}$ e $\mathrm{SiO}_{2}$, sendo o resíduo parcialmente amorfo com picos cristalinos. Ao submetê-lo ao aquecimento, ficou evidente que o lodo possui cerca de $20 \%$ de matéria orgânica. O lodo têxtil apresentou evidências positivas que reiteram a possibilidade de sua utilização como constituinte de materiais de construção alternativos.
\end{abstract}

Palavras-chave: Lodo têxtil; Resíduo industrial; Materiais alternativos; Materiais de construção.

\footnotetext{
Abstract

The textile sludge is a waste resulting of the physicochemical treatment procedure carried out in the effluent of textile laundries. This material is classified by the Brazilian standard as a non-hazardous waste considering that, despite its varied chemical composition, its components do not present great risks of human contamination. In view of the
} 
inadequate disposal of this waste, it was noticed the need to alternatives that allow the use of this material, since, currently, there is no recycling or use. The aim, therefore, was to verify that construction materials are an excellent matrix that receives this waste, providing a considerable reduction in its inadequate disposal and the possible environmental damage it generates. Specific mass analyzes, laser granulometry, thermogravimetry and differential thermal analysis, X-ray fluorescence, X-ray diffraction, scanning electron microscopy and infrared spectroscopy by Fourier transform were executed, using them to characterize the sludge. A value of $2.20 \mathrm{~g} / \mathrm{cm}^{3}$ was obtained for its specific mass and an average diameter of $26.10 \pm 0.65 \mu \mathrm{m}$, with $97.15 \%$ Regarding its composition, its main components are $\mathrm{CaO}, \mathrm{MgO}$ and $\mathrm{SiO}_{2}$, the waste being a partially amorphous material of them having a diameter less than $80 \mu \mathrm{m}$, what characterized the textile sludge as a filler. Subjecting the sample to heating, it was evident that it has about $20 \%$ organic matter. The textile sludge presented positive evidence that reiterates your use in alternative building materials. Keywords: Textile sludge; Industrial waste; Alternative materials; Construction materials.

\section{Resumen}

El lodo textil es un residuo resultante del procedimiento de tratamiento físico-químico realizado en el efluente de las lavanderías textiles. Este material está clasificado por la norma brasileña como residuo no peligroso considerando que, a pesar de su variada composición química, sus componentes no presentan grandes riesgos de contaminación humana. Ante el escenario actual de disposición inadecuada de estos residuos por parte de las lavanderías, percibió la necesidad de alternativas que permitan el uso de este material, ya que, actualmente, no hay reciclaje ni aprovechamiento. Por tanto, el objetivo era comprobar que los materiales de construcción son una excelente matriz que recibe estos residuos, proporcionando una reducción considerable de su disposición inadecuada y del posible daño ambiental que genera. El lodo se caracterizó mediante pruebas de masa específica, granulometría láser, termogravimetría y análisis térmico diferencial, fluorescencia de rayos X, difracción de rayos X, microscopía electrónica de barrido y espectroscopía infrarroja por transformada de Fourier. Se obtuvo un valor de $2,20 \mathrm{~g} / \mathrm{cm}^{3}$ para su masa específica, y un diámetro medio de $26,10 \pm 0,65 \mu \mathrm{m}$, siendo $97,15 \%$ de ellas de diámetro inferior a $80 \mu \mathrm{m}$, lo que lo caracteriza como filler. En cuanto a su composición, sus principales componentes son $\mathrm{CaO}, \mathrm{MgO}$ y $\mathrm{SiO}_{2}$, siendo el residuo un material parcialmente amorfo. Al someterlo a calentamiento, se evidenció que tiene alrededor de $20 \%$ de materia orgánica. El lodo textil presentó evidencias positivas que reiteran el éxito de su uso en materiales de construcción alternativos.

Palabras clave: Lodo textil; Residuos industriales; Materiales alternativos; Materiales de construcción.

\section{Introdução}

Na região Agreste de Pernambuco, é abrigado um dos maiores polos têxteis do Brasil, o APL (Arranjo Produtivo Local) de Confecções do Agreste. Seus principais membros são os municípios de Caruaru, Santa Cruz do Capibaribe e Toritama que, junto aos demais, representam o segundo maior volume de produção nacional. A produção local, principalmente o município de Toritama, destaca-se na manufatura do jeans. Seu processo de fabricação é composto por algumas etapas que, além das fábricas de confecção, também envolve um grande número de indústrias de beneficiamento, denominadas lavanderias industriais. Como afirmam Cometti et al. (2016), as lavanderias industriais são responsáveis pelos processos de lavagem, amaciagem, tingimento, descoloração e demais processos de modificação nas peças.

Segundo Souto (2017), o efluente proveniente das lavanderias carrega cerca de $90 \%$ dos produtos químicos empregados nos procedimentos realizados, os quais são de composição extremamente heterogênea, em decorrência da variedade de processos executados e dos materiais neles utilizados. Há registros de uma série de eventos nos quais rios de grande relevância para a região tiveram trechos totalmente contaminados pelos descartes irregulares.

Tendo em vista os danos ocasionados, percebeu-se a necessidade de implementar métodos específicos de tratamento, antecedendo o descarte em alguma reserva hídrica natural. A depender do objetivo do tratamento e da disponibilidade de recursos, existem diferentes processos a serem realizados, desde os de simples aplicação, como os físico-químicos, mais empregados na região Agreste, a alguns mais complexos, como tratamentos biológicos e processos oxidativos avançados (POA).

No estado de Pernambuco, por meio de esforços realizados pela Agência Estadual de Meio Ambiente (CPRH) e Ministério Público Estadual a partir de 2004, foram adotados programas de controle ambiental, incluindo o tratamento do efluente das lavanderias. Ainda hoje, muitas optam pelo procedimento mais simples, de coagulação-floculação, seguido de filtragem, baseado em métodos físico-químicos.

O processo de tratamento do efluente das lavanderias pode ser dividido em algumas etapas: gradeamento, no qual são 
retirados os resíduos maiores, como retalhos de tecido; equalização, por meio do qual o fluido passa por tanques para homogeneizar a mistura; coagulação/floculação/decantação, na qual o efluente é submetido a agentes coagulantes, sendo muito utilizados o policloreto de alumínio $\left(\mathrm{Al}_{2}(\mathrm{OH})_{3} \mathrm{Cl}_{3}\right)$ e o sulfato de alumínio $\left(\mathrm{Al}_{2} \mathrm{SO}_{4}\right)$; filtração, onde separa-se a porção líquida, descartada ou reutilizada, e a sólida, comumente descartada de forma inadequada.

Apesar desses processos reduzirem ou evitarem a contaminação das porções de água, o descarte precário do lodo pode ocasionar sérios danos ao solo, por exemplo. Segundo a Agência Estadual de Meio Ambiente (CPRH), o lodo têxtil, ou lodo industrial, é o principal resíduo sólido gerado pelas lavanderias e é considerado como resíduo não perigoso (Classe II), conforme a NBR 10004/2004. É sabido que o material, devido aos processos que regem sua formação, possui composição química variada, a depender da forma de processamento das peças de roupa e do tratamento do efluente.

Assim, com base na literatura, foi elaborada a Tabela 1, que apresenta composições do resíduo de diferentes localidades geográficas e, consequentemente, variados processos produtivos. Em sublinhado duplo são indicados componentes em maior concentração em cada amostra; para a segunda maior taxa, a indicação se dá pelo sublinhado simples; para a terceira, sublinhado pontilhado.

Tabela 1. Composição química de diferentes amostras de lodo têxtil.

\begin{tabular}{|c|c|c|c|c|c|c|c|c|c|c|}
\hline & 1 & 2 & 3 & 4 & 5 & 6 & 7 & 8 & 9 & 10 \\
\hline Origem & PE-BR & PB-BR & PB-BR & RJ-BR & SC-BR & SC-BR & SC-BR & Índia & China & Malásia \\
\hline $\mathrm{CaO}$ & $8,92 \%$ & $\underline{11,71 \%}$ & $\underline{10,52 \%}$ & $8.68 \%$ & $3,01 \%$ & $\underline{63,10 \%}$ & $24,51 \%$ & $\underline{\underline{33,50 \%}}$ & $0,94 \%$ & $2,14 \%$ \\
\hline $\mathrm{MgO}$ & - & 6.53\% & $5,86 \%$ & $1,11 \%$ & - & $6.30 \%$ & $0,51 \%$ & $1,00 \%$ & - & $4,86 \%$ \\
\hline $\mathrm{SiO}_{2}$ & $\underline{\underline{\mathbf{3 6}, 82 \%}}$ & $\underline{\mathbf{5 9 , 5 9 \%}}$ & $\underline{69,73 \%}$ & $\underline{41,93 \%}$ & $16,33 \%$ & $5,10 \%$ & $\underline{43,25 \%}$ & $3,80 \%$ & $2,50 \%$ & $\underline{49,29 \%}$ \\
\hline $\mathrm{SO}_{3}$ & 11,07\% & $3,51 \%$ & $5,55 \%$ & - & $2,52 \%$ & $4,10 \%$ & - & $0,40 \%$ & $\underline{18,23 \%}$ & $15,71 \%$ \\
\hline $\mathrm{Al}_{2} \mathrm{O}_{3}$ & $\underline{22,13 \%}$ & $2,51 \%$ & $0,92 \%$ & $\underline{37,81 \%}$ & $\underline{\underline{55,96 \%}}$ & $\underline{17,00 \%}$ & $\underline{25,23 \%}$ & $0,30 \%$ & $4.56 \%$ & $6,64 \%$ \\
\hline $\mathrm{MnO}$ & $1,55 \%$ & - & - & $1,12 \%$ & - & - & $0,10 \%$ & - & $0,60 \%$ & $4,57 \%$ \\
\hline $\mathrm{Fe}_{2} \mathrm{O}_{3}$ & $8,16 \%$ & $1,28 \%$ & $0,60 \%$ & $3,48 \%$ & $3,71 \%$ & $0,30 \%$ & $1,44 \%$ & $\underline{18,90 \%}$ & $\underline{44,51 \%}$ & $1,50 \%$ \\
\hline $\mathrm{Na}_{2} \mathrm{O}$ & - & - & $0,40 \%$ & $1,32 \%$ & - & $1,20 \%$ & $0,62 \%$ & $0,06 \%$ & - & $0,57 \%$ \\
\hline $\mathrm{K}_{2} \mathrm{O}$ & $3,49 \%$ & - & $1,05 \%$ & $1,38 \%$ & $1,42 \%$ & $0,40 \%$ & $2,37 \%$ & $0,04 \%$ & - & $1,00 \%$ \\
\hline $\mathrm{P}_{2} \mathrm{O}_{5}$ & $7,23 \%$ & $2,62 \%$ & $3,92 \%$ & $2,80 \%$ & $14,85 \%$ & $2,20 \%$ & $1,65 \%$ & - & $2,05 \%$ & $8,57 \%$ \\
\hline OUTROS & $0,63 \%$ & $12,25 \%$ & $5,37 \%$ & $0,37 \%$ & $2,20 \%$ & $0,30 \%$ & 0,32 & $42,00 \%$ & $26,61 \%$ & $5,15 \%$ \\
\hline
\end{tabular}

Fonte: Adaptado de (1) Leloup (2013); (2) Oliveira (2014); (3) Aquino et al. (2014); (4) Ferreira (2018); (5) Sonai et al. (2015); (6) Anjos (2017); (7) Zanoni (2001); (8) Goyal et al. (2019); (9) Zhan et al. (2019); (10) Aziz et al. (2017).

A quantidade de lodo gerado em uma lavanderia está relacionada à eficiência dos sistemas de tratamento de efluentes e aos métodos adotados, físico-químicos ou biológicos. Segundo Souto (2017), uma lavanderia industrial têxtil de médio porte, que processa 100 mil peças de vestuários por ano, pode gerar, aproximadamente, 6 toneladas de lodo têxtil seco nesse período. Dias Júnior (2013) afirma que, entre outros fatores, o descarte não consciente desse resíduo tornou-se um problema ambiental para as lavanderias têxteis.

Nesse sentido, buscando trazer soluções aos danos ambientais gerados, são cada vez mais frequentes os estudos que analisam o aproveitamento de resíduos de diversos segmentos industriais para incorporação em outros materiais, como os utilizados na engenharia civil.

Diversos autores já realizaram o estudo da incorporação de lodo têxtil para fins de imobilização em materiais da 
construção civil, tais como Herek et al. (2012), Zanoni (2013), Zhan e Poon (2015), Aquino et al. (2015), Velumani et al. (2015), Anwar et al. (2018), Hossain et al. (2018), Ferreira (2018), Zhan et al. (2019), Goyal et al. (2019) e Oliveira et al. (2020). Essa incorporação acontece em matrizes diversas tais como: argamassas, blocos cerâmicos, pavimentos, entre outros.

Conforme Aquino et al. (2015), a utilização do resíduo em blocos cerâmicos possibilitou a diminuição da retração linear; já Oliveira et al. (2020) constataram que o lodo apresenta ótimo desempenho na estabilização de camadas de base e subbase de pavimentos; Goyal et al. (2019) observaram que a adição de até 5\% de lodo na produção de argamassas de cimento não afetou negativamente o comportamento dos corpos de prova após o tempo de cura. Por meio da análise das cargas minerais utilizadas em polímeros (Gonçalves et al., 2019), a composição química, com altos teores de óxido de cálcio, sílica e alumina, e a granulometria descritas, são compatíveis ao lodo, direcionando-o potencialmente para essa finalidade.

A fim de concretizar o lodo têxtil como material alternativo para a construção civil, foram elaborados diversos ensaios que propiciaram a caracterização química e física do resíduo, sendo eles apresentados por meio desse trabalho.

\section{Metodologia}

\subsection{Coleta e preparação da amostra}

O lodo têxtil utilizado nas análises foi coletado no leito de secagem (Figura 1) de uma lavanderia de médio porte situada em Toritama, município do estado de Pernambuco. No leito de secagem, o lodo têxtil reduziu grande parte da sua umidade e se aglomerou em placas, sendo essa a última etapa do tratamento físico-químico.

Figura 1. Lodo têxtil no leito de secagem.

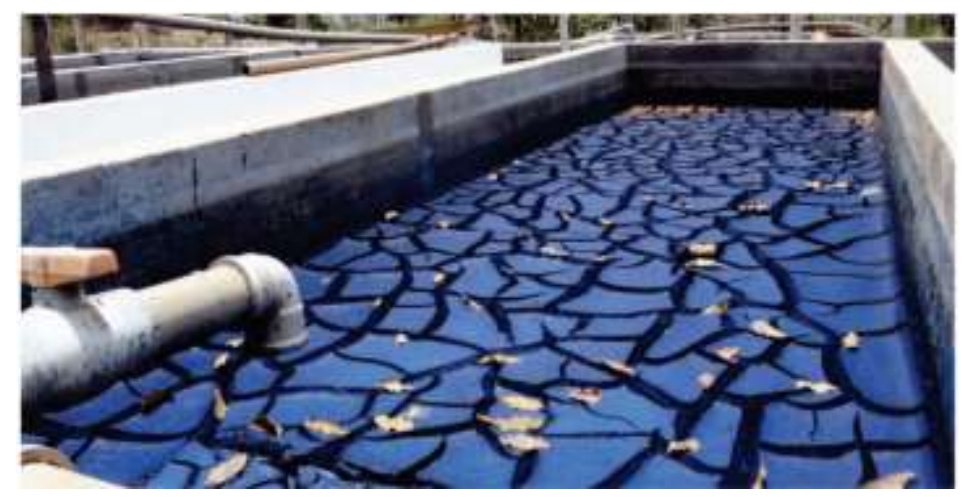

Fonte: Cartilha Lavando Limpo (2018).

Posteriormente, o resíduo foi submetido à secagem ao ar livre, sendo disposto sobre bandejas e, em seguida, levado à secagem completa, ao ser conduzido a uma estufa. Após esse processo, o lodo foi inserido em um moinho de barras de baixa energia, tendo como objetivo o destorroamento das partículas aglutinadas. Por fim, o material foi passado pela peneira \#200, de modo a assegurar a granulometria máxima de $75 \mu \mathrm{m}$ do material, possibilitando sua utilização enquanto fíler.

\subsection{Determinação da massa específica}

Para a obtenção da massa específica do lodo têxtil, foi utilizado frasco volumétrico de Le Chatelier, de acordo com a NBR 16605/2017.

\subsection{Granulometria a laser}

Foi utilizado o analisador do tamanho de partículas Microtac, modelo S3500, para a caracterização granulométrica do lodo têxtil, obtendo sua curva de granulometria para as partículas menores que $75 \mu \mathrm{m}$. 


\subsection{Análise termogravimétrica (TGA)/Termogravimetria derivada (DTG)}

Buscando acompanhar a variação de massa do resíduo em função de incrementos de temperatura, foi realizado o ensaio de termogravimetria, em atmosfera de ar, a uma taxa de aquecimento de $10{ }^{\circ} \mathrm{C} / \mathrm{min}$ até a temperatura de $900{ }^{\circ} \mathrm{C}$, utilizando um analisador térmico simultâneo da marca Netzsch, modelo STA 2500 Regulus.

\subsection{Fluorescência de Raios-X (FRX)}

A determinação dos elementos químicos presentes no lodo e suas respectivas proporções foi realizada com auxílio de um espectrofotômetro de fluorescência de raios-X, da marca Rigaku, modelo Primini.

\subsection{Difração de Raios-X (DRX)}

A caracterização qualitativa da estrutura cristalina do lodo têxtil foi realizada em um difratômetro de Raios-X, da marca Rigaku, modelo Miniflex 600, com radiação $\mathrm{CuK} \alpha(40 \mathrm{kV} / 30 \mathrm{~mA})$, sendo a velocidade do goniômetro de $0,02^{\circ} 2 \theta$ por passo com tempo de contagem de 1,0 segundo por passo e coletados de 10 a $80^{\circ} 2 \theta$.

\subsection{Microscopia Eletrônica de Varredura (MEV)}

A análise procedida via microscopia eletrônica de varredura foi realizada utilizando microscópio de varredura Shimadzu SSX550 SUPESCAR e metalizador, Shimadzu IC 50. As ampliações se deram em escalas de 1200 a 5000 vezes.

\subsection{Espectroscopia no Infravermelho por Transformada de Fourier (FTIR)}

Para identificar os grupos funcionais do lodo têxtil por meio da vibração dos átomos no infravermelho, foi realizado o ensaio de FTIR, utilizando um espectrofotômetro de FTIR da marca Shimadzu, modelo IRPrestige-21. Às amostras em pó foi incorporado $\mathrm{KBr}$, estas foram comprimidas em pastilhas e varridas na faixa espectral de 4000 para $400 \mathrm{~cm}^{-1}$.

\section{Resultados e Discussão}

\subsection{Determinação da massa específica}

A partir do experimento, aferindo o volume inicial e final, tendo por base a massa da amostra de lodo no frasco, foi possível obter o valor de massa específica de $2,20 \mathrm{~g} / \mathrm{cm}^{3}$. Valor que se assemelha à massa específica da cinza de casca de arroz, resíduo largamente inserido em materiais de construção civil, que possui cerca $2,16 \mathrm{~g} / \mathrm{cm}^{3}$, conforme Silva (2019).

\subsection{Granulometria a laser}

Por meio dos resultados, foi construída a curva granulométrica expressa na Figura 2. 
Figura 2. Granulometria a laser do lodo têxtil.

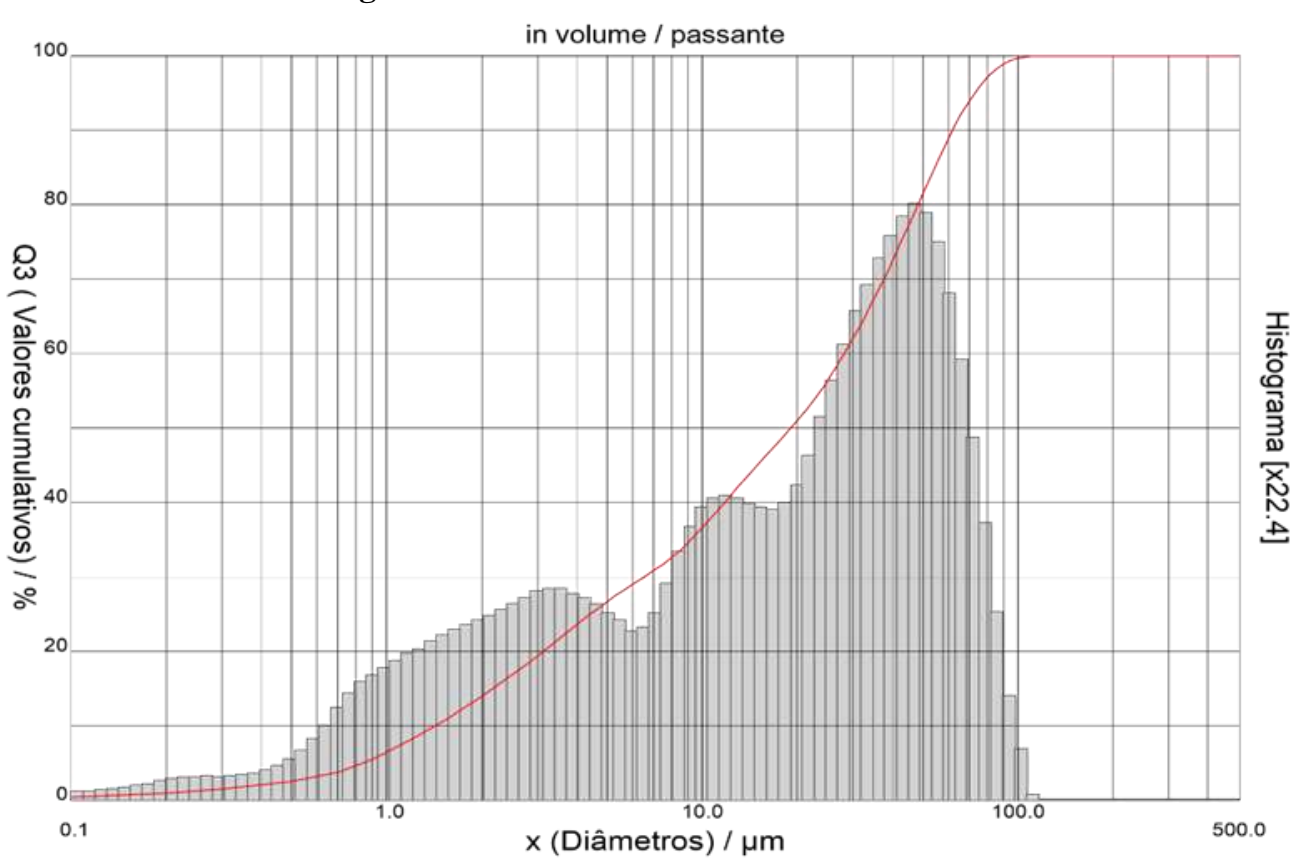

Fonte: Autores (2021).

De acordo a curva granulométrica, apresentada pela Figura 2, foi possível obter o diâmetro médio das partículas de lodo têxtil, sendo esse valor de $26,10 \pm 0,65 \mu \mathrm{m}$. Para os diâmetros médios a passantes $10 \%$, tem-se $1,42 \mu \mathrm{m}$; a $50 \%$, tem-se 19,05 $\mu \mathrm{m}$; por fim, para 90\%, tem-se $61,99 \mu \mathrm{m}$. Em uma análise geral, observa-se que $97,15 \%$ da porção do lodo têxtil possui diâmetro inferior a $80 \mu \mathrm{m}$. Dessa forma, as partículas atendem ao requisito mínimo de $65 \%$ passante na malha \#200, com abertura de 75 $\mu \mathrm{m}$, para ser considerado fíler (DNIT EM 367/97, 1997).

A importância do efeito fíler é abordada por Silva et al. (2019) e Pereira et al. (2021), que evidenciam como o aumento da área específica proporciona o melhor preenchimento dos vazios e, consequente, aumento da compacidade do material. Conforme a AASHTO (1991), misturas asfálticas, por exemplo, quando contêm partículas ultrafinas, menores que de $20 \mu \mathrm{m}$, possuindo alguma porção menor que $5 \mu \mathrm{m}$, tendem a compor uma película junto ao ligante, denominada mastique. Essa película envolve as partículas de agregados miúdos e graúdos e a razão do volume de ligante livre pelo volume total de ligante tem efeito significativo na rigidez do mastique (Motta \& Leite, 2000). Já para materiais cimentícios, especificamente para concreto, Damineli (2013) relata a utilização de fíler como uma ferramenta para aumentar o fator de empacotamento, aprimorando o comportamento reológico da mistura.

\subsection{Análise termogravimétrica (TGA)/Termogravimetria derivada (DTG)}

Na Figura 3, são apresentadas as curvas de variação da massa em função da temperatura, em preto, e a derivada primeira dessa variação em função do tempo, em vermelho. 
Figura 3. Termogravimetria do lodo têxtil.

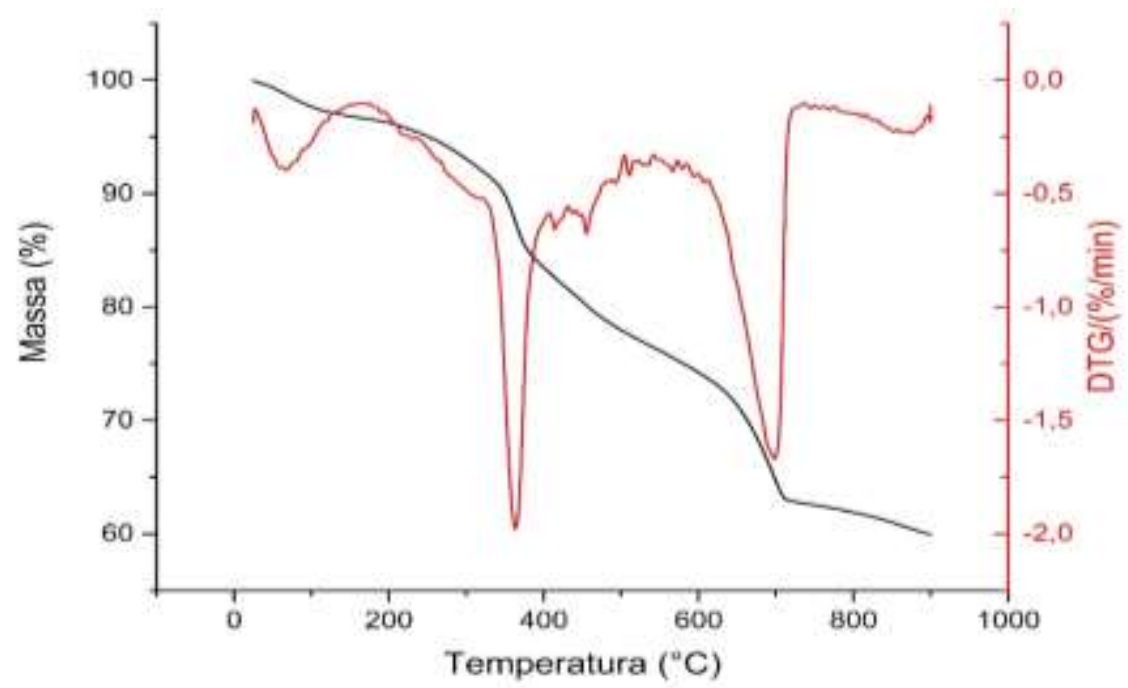

Fonte: Autores (2021).

$\mathrm{Na}$ análise da variação de massa em função da temperatura (DTG), podem ser observados, por meio do gráfico, dois estágios. O primeiro envolve uma perda de massa de cerca de $5 \%$, com temperatura máxima por volta de $50{ }^{\circ} \mathrm{C}$, relacionado com a umidade residual existente na amostra de lodo têxtil. O segundo estágio ocorre com temperatura máxima por volta de $350{ }^{\circ} \mathrm{C}$, com perda de cerca de $20 \%$, que corresponde à porção de matéria orgânica contida.

\subsection{Fluorescência de Raios-X (FRX)}

Como produto do ensaio de FRX e correção do percentual de matéria orgânica, obtido por meio da análise termogravimétrica, foi elaborada a Tabela 2, na qual é possível observar os percentuais dos principais óxidos constituintes do lodo têxtil. Sendo a porção de matéria orgânica cerca de $20 \%$, o total dos valores obtidos pelo equipamento, que não a mensura, representam cerca de $80 \%$.

Tabela 2. Composição química do lodo têxtil.

\begin{tabular}{cc|cc|cc}
\hline \multicolumn{7}{c}{ Componente / Percentual (\%) } \\
\hline $\mathrm{CaO}$ & 28,18 & $\mathrm{SO}_{3}$ & 4,34 & $\mathrm{MnO}$ & 1,63 \\
$\mathrm{MgO}$ & 20,98 & $\mathrm{Al}_{2} \mathrm{O}_{3}$ & 3,62 & $\mathrm{Fe}_{2} \mathrm{O}_{3}$ & 1,44 \\
$\mathrm{SiO}_{2}$ & 14,81 & $\mathrm{Cl}$ & 2,18 & Orgânicos* & 20,00 \\
\hline
\end{tabular}

*Valor obtido pelo TGA/DTG ** Outros 2,82\%. Fonte: Autores (2021).

A partir das informações químicas do lodo estudado, é possível construir uma correlação entre os processos de diferenciação do jeans e do tratamento realizado no efluente. Ao observar a tabela apresentada, é evidente que a sílica tem altas concentrações no lodo industrial, assim como as amostras analisadas na Tabela 1, por meio da qual se observa que esse é o elemento de maior ou segunda maior taxa de composição em oito das dez amostras. Tal aspecto é decorrente da utilização da argila expandida (que possui como principal componente a sílica $\left(\mathrm{SiO}_{2}\right)$ ), conforme Moravia (2006), na estonagem, um processo que desgasta o tecido por motivos estéticos e o prepara para receber os alvejantes e corantes. Esse procedimento também é conhecido por stone washed, ou seja, "lavadas à pedra", como apresentam Silva Filho et al. (2021).

Outro componente obtido é o óxido de alumínio $\left(\mathrm{Al}_{2} \mathrm{O}_{3}\right)$, que está diretamente relacionado ao processo de tratamento físico-químico, no qual se emprega sulfato ou policloreto de alumínio na etapa de floculação. Este é identificado em altas 
concentrações nas amostras (1) Leloup (2013), (4) Ferreira (2018), (5) Sonai et al. (2015), (6) Anjos (2017) e (7) Zanoni (2001), por meio da Tabela 1. Soluções férricas também são utilizadas para promover a solubilização, fica evidente essa substituição nas amostras (8) Goyal et al. (2019 e (9) Zhan et al. (2019), que apresentam um teor elevado de óxido de ferro $\left(\mathrm{Fe}_{2} \mathrm{O}_{3}\right)$, também obtido na análise do lodo estudado (Tabela 2), e baixa concentração de óxido de alumínio $\left(\mathrm{Al}_{2} \mathrm{O}_{3}\right)$.

É possível observar certa variabilidade da amostra estudada por Leloup (2013), também oriunda do APL do Agreste pernambucano, o que enfatiza que as lavanderias podem adotar processos produtivos e de tratamento distintos, o que pode conferir variação sazonal ou permanente da composição do lodo.

\subsection{Difração de Raios-X (DRX)}

Foi gerado, a partir do ensaio de DRX, o difratograma de raios-X, ilustrado na Figura 4.

Figura 4. Difratograma de raios-X.

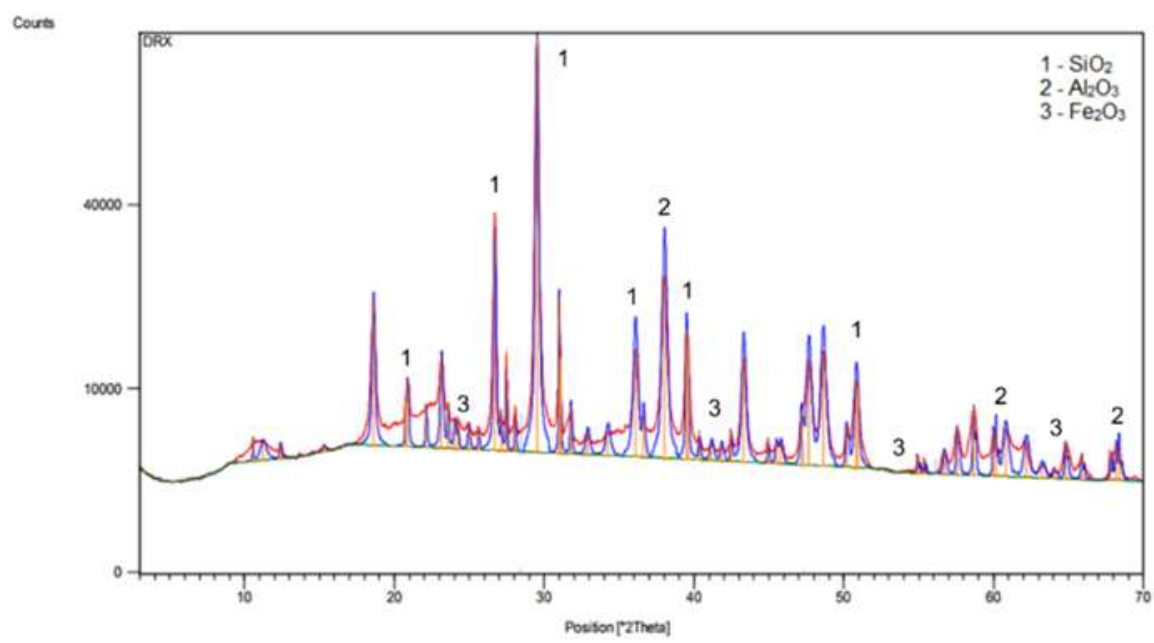

Fonte: Autores (2021).

No gráfico, pode-se verificar que o lodo têxtil contém material amorfo, devido ao formato de halo da curva. No entanto, pode ser constatada a presença de materiais cristalinos como o quartzo, alumina e óxido de ferro, indicados na Figura 4 na legenda do Gráfico por 1, 2 e 3, respectivamente.

\subsection{Microscopia Eletrônica de Varredura (MEV)}

As capturas efetuadas pela MEV podem ser observadas na Figura 5.

Figura 5. Lodo têxtil ampliado em 1200x (à esq.) e 5000x (à dir.).

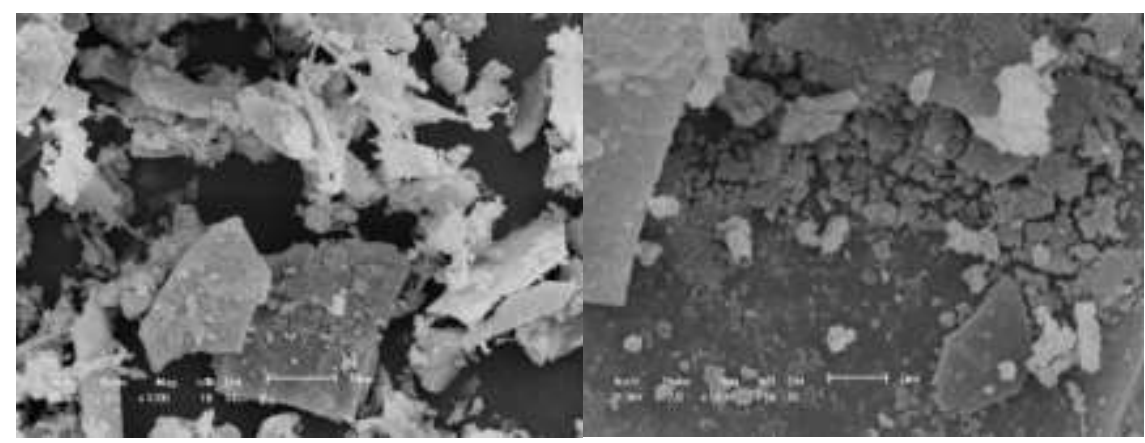

Fonte: Autores (2021). 
Por meio da análise da Figura 5, observa-se que, quanto ao tamanho e à forma das partículas do lodo têxtil, pode-se inferir que ele apresenta forma irregular e grãos com tamanhos diferenciados, entretanto as imagens revelam a existência de partículas ultrafinas.

\subsection{Espectroscopia no Infravermelho por Transformada de Fourier (FTIR)}

$\mathrm{Na}$ análise por espectroscopia no infravermelho com transformada de Fourier, a Figura 6 apresenta as bandas de absorção no infravermelho do lodo têxtil seco (seco em estufa), em preto, e úmido (ambiente), em vermelho. Os picos ocorrem nos comprimentos de onda $3400 \mathrm{~cm}^{-1}, 1650 \mathrm{~cm}^{-1}, 1040 \mathrm{~cm}^{-1}, 880 \mathrm{~cm}^{-1} \mathrm{e} 460 \mathrm{~cm}^{-1}$.

Figura 6. Espectroscopia no infravermelho com transformada de Fourier do Lodo Têxtil.

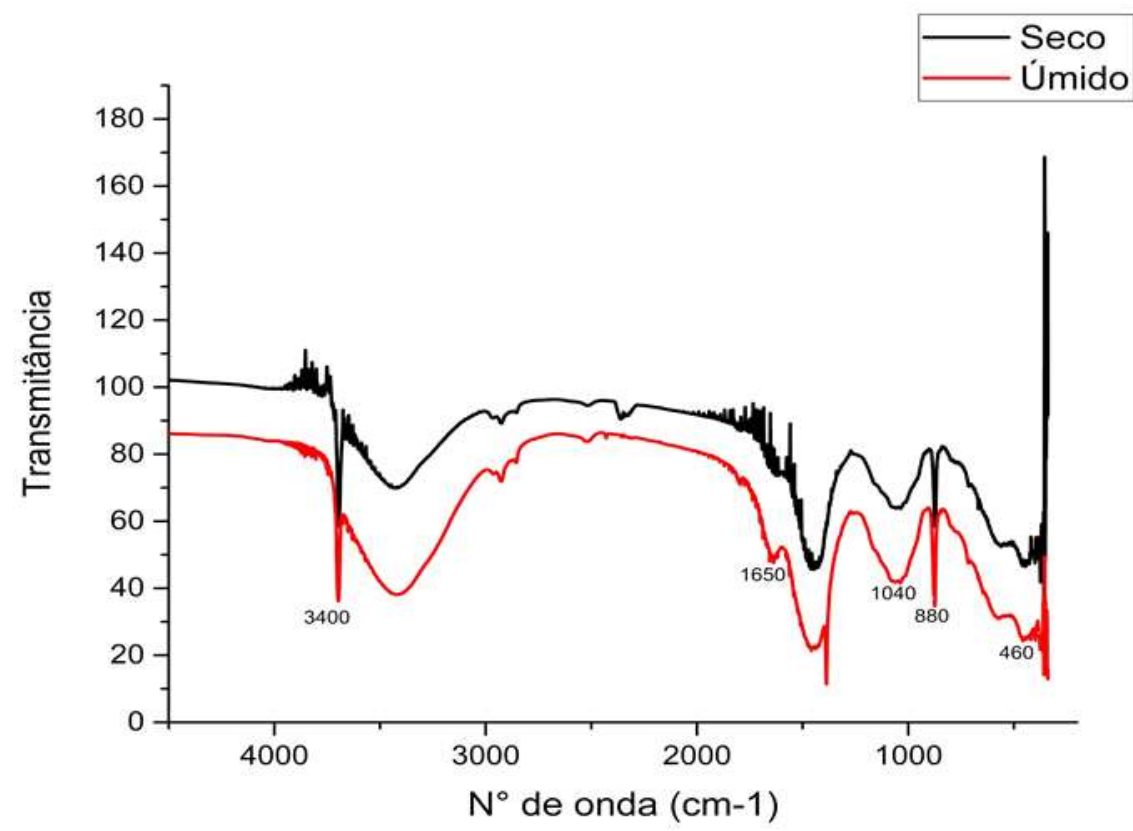

Fonte: Autores (2021).

A primeira observação que pode ser realizada é que, apesar da variação da transmitância entre o lodo seco e úmido, os valores de número de onda nos quais ocorreram os picos foram coincidentes. Assim, como o aspecto importante para a análise são esses pontos, a avaliação pode se dar de forma conjunta.

De acordo com o quadro exposto por Lima (2018), o pico em $3400 \mathrm{~cm}^{-1}$ indica ligações do tipo O-H; em $1650 \mathrm{~cm}^{-1}$, vibrações nas ligações H-O-H; em $1040 \mathrm{~cm}^{-1}$, vibrações de tensão assimétrica (Si-O-Si e Al-O-Al); $880 \mathrm{~cm}^{-1}$, tensão assimétrica Al-O; por fim, em $460 \mathrm{~cm}^{-1}$, tensão simétrica Al-O-Si, ratificando os resultados obtidos no ensaio de FRX.

\section{Conclusão}

Por meio dos resultados obtidos, foi possível caracterizar o lodo têxtil gerado na região Agreste de Pernambuco. Ficou evidente que esse material, após um simples processo de secagem e destorroamento, possui características físicas e químicas que sugerem um comportamento adequado enquanto componente de materiais de construção alternativos. $\mathrm{O}$ efeito fíler que ele ocasiona, em decorrência dos pequenos diâmetros das partículas que o compõem, é relevante no preenchimento de vazios das misturas compósitas, levando ao potencial de melhoras de desempenho. Suas características possibilitaram ainda, a boa estabilização de solos, a redução da retração linear em blocos cerâmicos e a não geração de danos mecânicos em corpos de prova de argamassa de cimento. Devido à sua composição química, associada à granulometria, também se observa um potencial uso 
como carga mineral em polímeros. Em aplicações práticas do resíduo, recomenda-se a realização de ensaios periódicos de TGA/DTG e FRX, a fim de analisar as possíveis alterações na sua composição química. Para trabalhos futuros, é sugerido que sejam estudados diferentes materiais de construção com adição de lodo têxtil, consequentemente também devem ser realizadas avaliações ambientais em relação aos materiais produzidos, para que, ao ser inserido em alguma matriz, seja ela cerâmica, cimentícia ou asfáltica, haja certificação da sua imobilização.

\section{Agradecimentos}

Os autores agradecem à Fundação de Amparo à Ciência e Tecnologia do Estado de Pernambuco (Facepe), à Pró-Reitoria de Pesquisa e Inovação (Propesqi) da Universidade Federal de Pernambuco e ao CNPq (Conselho Nacional de Desenvolvimento Científico e Tecnológico) pelas bolsas de iniciação científica.

\section{Referências}

Agência Estadual de Meio Ambiente. (2018). Lavando Limpo.

American Association of State Highway and Transportation Officials. (1991). Handbook of hot-mix asphalt paving.

Anjos, D. C. dos. (2017). Estudo sobre a influência do lodo têxtil gerado por uma lavanderia industrial, aplicado em argamassa de cimento Portland composto de filer. Universidade Federal de Santa Catarina.

Anwar, T. B., Behrose, B. \& Ahmed, S. (2018). Utilization of textile sludge and public health risk assessment in Bangladesh. Sustainable Environment Research, $1-6$.

Aquino, R. C. (2015). Adição de resíduo de lodo da indústria têxtil na produção de blocos cerâmicos de vedação. Revista Eletrônica de Materiais e Processos, $10,29-35$.

Associação Brasileira de Normas Técnicas. (2004). NBR 10004: Resíduos sólidos - Classificação.

Associação Brasileira de Normas Técnicas. (2017). NBR 16605: Cimento Portland e outros materiais em pó — Determinação da massa específica.

Aziz, H. A., Ghazali, M. F., Omran A. \& Umar M. (2017). Solidification and stabilization of the incinerated wastewater sludge from textile industry. AIP Conference Proceedings.

Cometti, J. L. S.; Silva, F. L.; Santos, F. J. H. \& Lima, V. A. (2016). Diagnóstico ambiental comparativo entre 2014 e 2015 das indústrias têxteis do município de Toritama-PE. Congresso Brasileiro de Gestão Ambiental.

Damineli, B. L. (2013). Conceitos para formulação de concretos com baixo consumo de ligantes: controle reológico, empacotamento e dispersão de partículas. Universidade de São Paulo.

Departamento Nacional de Infraestrutura de Transportes. (1997). DNIT-ME 367/97: Material de enchimento para misturas betuminosas.

Dias Júnior, M. L. (2013). Incorporação de lodo têxtil em blocos cerâmicos. Universidade Tecnológica Federal do Paraná.

Ferreira, M. das N. (2018). Incorporação de lodo de estação de tratamento de efluentes (ETE) de lavanderia industrial em cerâmica vermelha. Universidade Estadual do Norte Fluminense.

Gonçalves, F. M.; Torres, J. W. M.; Pascoal, A. L.; Ferreira, T. E. D.; Ferreira, E. E. \& Alexandrino, J. S. (2019). Aproveitamento do rejeito de lavra de ardósia para a produção de carga mineral. Holos Environment, 19 (2), 235-242.

Goyal, S., Rafat S., Sujant J. \& Devender S. (2019). Utilization of textile sludge in cement mortar and paste. Construction and Building Materials, 214, 169177.

Herek, L. C. S., Hori, C. E., Reis, M. H. M., Mora, N. D., Tavares, C. L. G. \& Bergamasco, R. (2012). Characterization of ceramic bricks incorporated with textile laundry sludge. Ceramics International, 38, 951-959.

Hossain, M. S., Das, S. C., Islam, J. M.M. \& Mamun, M. A. A. (2018). Reuse of Textile Mill ETP Sludge in Environmental Friendly Bricks - Effect of Gamma Radiation. Radiation Physics and Chemistry, 151, 77-83.

Leloup, W. D. A. (2013). Efeitos da adição de lodo têxtil e cinzas de lenha gerados no APL de confecções pernambucano em argamassas de cimento Portland. Universidade Federal de Pernambuco.

Lima, J. (2018). Efeito da relação molar $\mathrm{SiO}_{2} / \mathrm{Al}_{2} \mathrm{O}_{3}$ e nas condições de cura nas propriedades de geopolímeros obtidos com silicato de cinza de casca de arroz. Universidade Federal de Pernambuco.

Moravia, W. G.; Oliveira, C. A. S.; Gumieri, A. G. \& Vasconcelos, W. L. (2006). Caracterização microestrutural da argila expandida para aplicação como agregado em concreto estrutural leve. Cerâmica, 52, 193-199. 
Research, Society and Development, v. 10, n. 4, e40910414078, 2021

(CC BY 4.0) | ISSN 2525-3409 | DOI: http://dx.doi.org/10.33448/rsd-v10i4.14078

Motta, L. M. G., \& Leite, L. F. M. (2000). Efeito do fíler nas características mecânicas das misturas asfálticas [Filler effects on aphalt mixtures]. Portuguese.] In Proc., 11 Congresso Panamericano, 127, 1007-1017.

Oliveira, A. G. de. (2014). Estudo das propriedades mecânicas do lodo têxtil para fins de utilização em pavimentos rodoviários. Universidade Federal de Campina Grande.

Oliveira, A. G.; Barros, A. D.; Lucena, L. C. F. L.; Lucena, A. E. F. L. \& Patricio, J. D. (2020). Evaluation of calcined textile sludge as a stabilizing material for highway soil. Journal of Traffic and Transportation Engineering (English Edition), 7 (5), 688-699.

Pereira, A. G.; Vieira, C. S.; Oliveira, M. B.; Paiva, C. A. \& Silva, R. L. (2021). Avaliação laboratorial de misturas asfálticas à quente produzidas com grafite cominuído. Research, Society and Development, 10 (2), e25110212030.

Silva, L. R.; Gama, K. N. C.; Salles, P. V. \& Braga, F. C. S. (2019). Concreto com cinza de casca de arroz (CCA) e resíduos de construção e demolição (RCD). Research, Society and Development, 8 (4), e2684861.

Silva Filho, A. R. A.; Duarte, A. D.; Sinesio, E. P.; Silva, G. L. \& Pessôa, S. G. S. (2021). Classification, characterization and diagnosis of Jeans Processing Laundries in the City of Caruaru-PE, in Agreste Pernambucano. Research, Society and Development, 10 (1), e57810112186.

Sonai, G. G.; Souza, S. M. A. G. U.; Oliveira, D. \& Souza, A. A. U. (2015). The application of textile sludge adsorbents for the removal of Reactive Red 2 dye. Journal of Environmental Management, 168, 149-156.

Souto, T. J. (2017). Estudo do comportamento químico e ambiental de efluentes industriais e resíduos sólidos oriundos de lavanderias do Polo têxtil no Agreste pernambucano. Universidade Federal de Pernambuco.

Velumani, P., SenthilKumar, S. \& Premalatha, P. V. (2016). An Innovative Approach to Evaluate the Performance of Sludge-Incorporated Fly Ash Bricks. Journal of Testing and Evaluation, 44, 2155-2163.

Zanoni, S. M. (2013). Reutilização de resíduos de indústria têxtil como matéria-prima em cerâmica vermelha. Universidade do Extremo Sul Catarinense.

Zhan B. J. \& Poon C. S. (2015). Study on feasibility of reutilizing textile effluent sludge for producing concrete blocks. Journal of Cleaner Production, 101, 174-179.

Zhan B. J., Jiang-Shan L., Xuan D. X. \& Poon C. S. (2019). Recycling hazardous textile effluent sludge in cement-based construction materials: Physicochemical interactions between sludge and cement. Journal of Hazardous Materials. 